\title{
Changes in Ocular Surface Disease Evaluated after Nonpenetrating Deep Sclerectomy
}

Carlota Fuente-García ( $\nabla$ carlotafuentegarcia@gmail.com )

Ramon y Cajal University Hospital: Hospital Universitario Ramon y Cajal

Francisco José Muñoz-Negrete

Ramon y Cajal University Hospital: Hospital Universitario Ramon y Cajal

Elisabet de Dompablo-Ventura

Ramon y Cajal University Hospital: Hospital Universitario Ramon y Cajal

Javier Moreno-Montañés

University of Navarra: Universidad de Navarra

Gema Rebolleda

Ramon y Cajal University Hospital: Hospital Universitario Ramon y Cajal

\section{Research Article}

Keywords: glaucoma, Keratograph, non-penetrating deep sclerectomy ocular hyperaemia, ocular surface disease

Posted Date: August 30th, 2021

DOI: https://doi.org/10.21203/rs.3.rs-845361/v1

License: (c) (1) This work is licensed under a Creative Commons Attribution 4.0 International License. Read Full License 


\section{Abstract}

Objective: To analyse the ocular surface changes in eyes after the withdraw of anti-glaucomatous drugs when non-penetrating deep sclerectomy (NPDS) is performed.

Methods: 31 patients (33 eyes) diagnosed of glaucoma that underwent NPDS, were included in this prospective study. Control group included 33 eyes of 33 age and sex-matched volunteers. Five variables were studied with Keratograph $5 \mathrm{M}(\mathrm{K} 5 \mathrm{M})$ : ocular hyperaemia $(\mathrm{OH})$, non-invasive tear film break-up time ( $\mathrm{NI}-$ BUT), lower tear meniscus height (LTMH) and meibography. LTMH was also measured using the anterior segment module of Spectralis Fourier-Domain Optical Coherence Tomography (FD-OCT). Also, an evaluation of corneal and conjunctival staining was performed.

In addition, patients were asked to answer two questionnaires: Ocular Surface Disease Index (OSDI) and National Eye Institute Visual Functioning Questionnaire (NEI VFQ-25) before and 6-months after surgery.

Results: Before NPDS, treated eyes, showed worse objective data than healthy controls ( $\mathrm{p} \leq 0.049)$. In this group, a significant improvement was observed in the questionnaires ( $p<0.001)$, LTMH FD-OCT ( $p=0.037)$ and LTMH K5M ( $p=0.025), \mathrm{K} 5 \mathrm{M} \mathrm{OH}(\mathrm{p}=0.003)$, NI-BUT ( $=0.022)$ and conjunctival and corneal staining $(\mathrm{p}<0.001)$. No significant differences were observed between groups in FD-OCT and K5M LTMH, NI-BUT, corneal-conjunctival staining and in the most OH sector values at 6 months $(p \geq 0.62)$.

Conclusion: A significant improvement in the ocular surface was observed 6 months after NPDS, suggesting that the withdrawn of the topical antiglaucomatous treatment has a beneficial effect.

\section{Introduction}

Primary open-angle glaucoma (POAG) is a chronic, progressive, and irreversible multifactorial optic neuropathy that is characterized by changes in visual field and in optic nerve $(\mathrm{ON})$ head $(1,2)$. Elevated intraocular pressure $(\mathrm{IOP})$ is a major risk factor for the development and progression of glaucoma.(3)

Nowadays, the only strategy that has reliably demonstrated preservation of visual function is the reduction of IOP. In most cases, the initial therapy is medical treatment with topical hypotensive drugs that are maintained for long periods of time. Surgical and laser treatment continue to play an important role in the treatment of these patients when drugs fail (contraindications, side effects, lack of compliance...), the target pressure cannot be achieved or glaucoma progression is detected. $(4,5)$

In terms of surgical treatments, trabeculectomy and non-penetrating deep sclerectomy (NPDS) procedures reduce IOP allowing aqueous humor drainage into the subconjunctival space. Among the advantages of NPDS, it is worth mentioning that the progressive filtration of aqueous humor from the anterior chamber to the subconjunctival space reduces postoperative complications (6).

The term ocular surface disease (OSD) includes a wide spectrum of conditions such as dry eye syndrome (DES), anterior blepharitis, meibomian gland dysfunction (MGD), conjunctivitis and keratitis (7).

A higher prevalence of OSD has been described in glaucoma patients and has multifactorial etiology. As a comorbidity of glaucoma, OSD is influenced by the age, ethnicity, sex of the patient and chronic use of topical anti-glaucomatous drugs $(8,9)$. Furthermore, OSD has been responsible for poor adherence to treatment and a worse quality of life in glaucoma patients $(10,11)$.

Although there are several publications that describe changes in the ocular surface in patients with IOP lowering eye drops (8, 12-16), there are few studies that evaluate these changes after trabeculectomy $(17,18)$; and, to our knowledge there are no studies evaluating the changes observed in the ocular surface after NPDS.

Therefore, the purpose of this study is to evaluate the changes in OSD in patients with glaucoma after NPDS and the withdrawn of antiglaucomatous drugs. The OSD was also compared with healthy subjects (control group).

\section{Materials And Methods}

For this prospective study, a total of 31 POAG patients (33 eyes) who underwent NPDS for uncontrolled intraocular pressure (IOP) despite medical treatment, were consecutively recruited from the Glaucoma Unit (Hospital Universitario Ramón y Cajal, Madrid, Spain). 33 eyes of 33 healthy volunteers, age and sexmatched to the POAG group, were included as a control group.

The study was approved by the Clinical Research Ethics Committee of Ramon y Cajal Hospital (CEIC), and conducted in accordance with the principles of the Declaration of Helsinki. Before recruitment, written informed consent was obtained from each participant.

Inclusion criteria in POAG group were: age over 18 years, a minimum of six-month treatment period with topical hypotensive agents, clinical indication of NPDS, and best-corrected visual acuity (BCVA) $>0.3$ in the Snellen scale. Subjects were excluded in both groups if they had undergone ocular surgery (cataract, glaucoma, refractive, ocular surface surgery) within the last six months, use of contact lenses, ocular surface abnormalities (conjunctival chalasis, conjunctival scars and corneal irregularities), diagnosis of dry eye disease (according to the diagnostic criteria established in the Dry Eye WorkShop II (DEWS II)(19), known systemic diseases associated with dry eye (Sjögren's syndrome, rheumatoid arthritis, lupus erythematosus ...) or diagnosis of secondary glaucoma (congenital, traumatic, steroidal, uveitic). 
All participants underwent a standard examination including a general anamnesis, sex and medical history data, recording the age of each patient. Treatment duration (time since the onset of the disease), total daily eye drops, total daily eye drops with preservatives, type of active principles as well as the type of preservative (polyquaternium or benzalkonium chloride), daily preservative concentration (DPC) and cumulative preservative concentration (CPC) were recorded. Daily preservative concentration was calculated regarding the preservative concentration on each medication, provided in the product data sheet $(\mu \mathrm{g} / \mu \mathrm{L})$. The latter is then multiplied by 7 , as the average tear volume $(7 \mu \mathrm{L})$ remaining in the eye following the instillation of a single drop $(12)$. Finally, we multiply the DPC by the number of daily eye drops for each drug. In the case of patients with polytherapy, the following DPCs for each drug were added. Cumulative preservative concentration was determined as the product of DPC plus treatment duration (in days).

Anti-glaucoma eye drops were immediately removed after surgery, and patients started a combined treatment Tobramycin + dexamethasone (Tobradex ${ }^{\mathrm{TM}}$, Alcon Cusi, Barcelona, Spain) five times daily, in a descending pattern every week and Ketorolac (Acular ${ }^{\mathrm{T}}$, Allergan Pharmaceuticals Ireland, Westport, Ireland) twice a day for two months. All sutures were removed a month after surgery. No artificial tears or anti-glaucomatous drugs were prescribed within six months after NPDS.

OSD indicators were recorded in the following order by the same examiner: Ocular Surface Disease Index (OSDI) and National Eye Institute Visual Functioning Questionnaire (NEI VFQ-25); K5M and FD- OCT using the anterior segment module. In a separate room, slit-lamp (SL) examination was performed. Both areas presented similar environmental conditions heating to a temperature of $21-25^{\circ} \mathrm{C}$ with a weak source of ambient light, and with no ventilation ducts over the equipment being used. The schedule in which the different explorations were made was from 8 to 15 hours.

\subsection{Ocular Surface Disease Index (OSDI) and National Eye Institute Visual Functioning Questionnaire (NEI VFQ-25)}

All patients included in both groups answered two questionnaires: OSDI and NEI VFQ-25.

The OSDI has been reported to be reliable and effective to discriminate among stages of dry eye severity (20) and was validated in Brazil by Prigol et al. (21).It is composed of a 12-item questionnaire that is divided into 3 subscales: the first one is related to visual function (questions 1 to 5 ), the second one is associated with ocular symptoms (questions 6 to 9) and the third one regards environmental triggers (questions 10 to 12). All items of the OSDI questionnaire were scored from 0 to 4 -point Likert scale ( 0 to 4 corresponding to: none of the times, some of the times, half of the time, most of the time, and all of the time, respectively). This total score was recorded for each participant. The impact of dry eye was then assessed categorically as normal (scores of $0-12$ ), mild (scores of 13-22), moderate (scores of 23-32), or severe (scores of 33-50), as previously described (15).

Patients also answered to the NEI VFQ-25 questionnaire, which was validated in Brazil by Simao et al. (22). NEI VFQ 25 measures the dimensions of selfreported vision-targeted health status, which concerns above all persons who have chronic eye diseases. We used the version that is most commonly used in studies including glaucoma patients. It consists of 12 subscales as follows: general health; general vision; difficulties with distance-vision activity; visionspecific role difficulties; peripheral; difficulties with near-vision activities; colour vision; vision-related dependency; mental health; social function; ocular pain; and driving difficulties. Each subscale consists of a minimum of one item and a maximum of four items. The algorithm has a scale ranging from 0 to 100 , with higher scores representing a better visual function.

OSDI and NEI VFQ 25 were administered to all subjects at the beginning of the session. In POAG group, we repeated the questionnaires six months after surgery.

\subsection{Oculus Keratograph $5 \mathrm{M}(\mathrm{K} 5 \mathrm{M})$}

Oculus Keratograph 5M (K5M) (Oculus Optikgerate GmbH, Wetzlar, Germany) is a device that consists of a keratometer and an optimized colour camera. The TF-Scan module was used to record non-invasive tear film break-up time (NI-BUT) and to measure low tear meniscus height (LTHM), through infrared lighting. The instrument was aligned at the pupil centre and NI-BUT measurements started when the patient blinked. The device shows two NI-BUT values: the first break-up time (NIBUTf) and average (NIBUTav). For LTHM measurements, the scan was taken below the corneal vertex, centered on the inferior cornea and lower eyelid. We used the integrated calliper tool provided by the device.

Through the R-Scan system, K5M allows us to perform an automatic classification of conjunctival redness. The patients were required to stare straight ahead and focus on the fixation mark inside the camera, so that the entire corneal area appeared in the center of the image. After generating a keratograph image, five redness scores (RS) (accurate to within 0.1 unit) are displayed on the computer screen. These scores (nasal limbal, temporal limbal, nasal bulbar, temporal bulbar and global) appear along with the total area analyzed $\left(\mathrm{mm}^{2}\right)$.

Meibography was assessed with upper and lower eyelid eversion using the meibography tool to generate IR images of tarsal conjunctiva. Manual grading of these images was performed using a meiboscore scale: grades from 0 to 3 ; being, no loss of meibomian glands: grade 0 ; loss of less than $1 / 3$ of the total meibomian gland area: grade 1 ; loss of $1 / 3$ to $2 / 3$ of the total area: grade 2 and loss more than $2 / 3$ of the area grade 3 (23).

\subsection{Spectralis Fourier-domain optical coherence tomography (FD-OCT)}

The anterior segment module of Spectralis Fourier-domain optical coherence tomography (Heidelberg Engineering GmbH, Heidelberg. Germany ) was used to measure LTMH using the method described by Arriola-Villalobos et al. (24). Briefly, the lower meniscus was imaged using the anterior segment lens with the image capture software in the sclera mode, high speed, and single vertical scan. The scan was taken below the corneal vertex, centered on the inferior cornea and lower eyelid. The corneal vertex was determined by the position of specular reflection. The patient was asked to blink normally during the imaging procedure while looking straight at a fixation target. Images were captured within the first second immediately after the blink. For LTMH measurements on the images, we used the calliper tool provided by the gadget. 


\subsection{Slit-lamp examination and corneal-conjunctival staining}

All subjects underwent a standard slit-lamp (SL) examination at A16 magnification in the second office. The cornea and the conjunctiva were stained using one fluorescein drop (Minims-fluorescein sodium 2.0\%; Chauvin Pharmaceuticals Ltd., Surrey, UK) to detect corneal and conjunctiva injury. Both were graded from zero to five using the Oxford scale ( 0 = no staining; I = minimum; II = mild; III = moderate; IV = marked; $V=$ severe) (25).

\subsection{Statistical analysis}

All statistical tests were performed using IBM SPSS version 15.0 (SPAA Inc., Chicago, IL.)

The qualitative variables were described in percentages, and quantitative variables were described with means and standard deviations or median, maximum, and minimum depending on their distributional characteristics in both groups. Kolmogorov-Smirnov test was used to assess the normal distribution of data.

Paired sample T-test was used for the comparative analysis on the repeated measurements in POAG group or its corresponding non-parametric Wilcoxon test for non-normal data. All contrasts were bilateral with a significance level of 0.05 .

For the comparative analysis of the different measurements between POAG group and controls, the Student's T test for independent samples or its corresponding non-parametric $U$ of Mann-Whitney test was used. All contrasts were bilateral with a significance level of 0.05 .

\section{Results}

The data analysed derived from 33 eyes with POAG and 33 healthy control eyes. Mean age was $75.33 \pm 7.5$ years in POAG group and $72.91 \pm 7.3$ years in controls $(p=0.188)$.

The mean duration of treatment from the start date was $108 \pm 309$ months. $87.87 \%$ of our patients were receiving three or more drops per day, while $66.67 \%$ received three or more preservative drops per day. $100 \%$ of our patients used at least one drop of preservative, being benzalkonium chloride (BAK) the most frequently used.

In the POAG group, $84,85 \%$ of patients were receiving prostaglandines. In the same line, $84,85 \%$ received $\boldsymbol{\beta}$-Blocker, $72,72 \%$ carbonic anhydrase inhibitor, and $48,48 \%$ adrenergic agonist. The mean of DPC and CPC was $1.89 \pm 0.88$ and $1670.15 \pm 1973.48$, respectively. Table 1 summarizes clinical, demographic and therapy data. 
Table 1

Clinical, demographic and treatment data.

\begin{tabular}{|c|c|c|c|}
\hline Parameters & POAG GROUP & CONTROL GROUP & P value \\
\hline SEX N (\%) & & & $p=0.624 * \star$ \\
\hline Female & $18 / 33(54.54 \%)$ & $17 / 33(51.51 \%)$ & \\
\hline Male & $15 / 33(45.45 \%)$ & $16 / 33(48.48 \%)$ & \\
\hline AGE (years +/- SD) & $75.33 \pm 7.5$ & $72.91 \pm 7.3$ & $p=0.188^{*}$ \\
\hline \multicolumn{4}{|c|}{ Treatment duration (months) } \\
\hline Median +/-SD & $108 \pm 309$ & & \\
\hline Minimum & 2 & & \\
\hline Maximun & 1792 & & \\
\hline \multicolumn{4}{|l|}{ DAILY EYEDROPS N (\%) } \\
\hline 1 & $3(9,09 \%)$ & & \\
\hline 2 & $1(3,03 \%)$ & & \\
\hline$\geq 3$ & $29(87,87 \%)$ & & \\
\hline \multicolumn{4}{|c|}{ DAILY EYEDROPS WITH PRESERVATIVES N (\%) } \\
\hline 1 & $4(12,12 \%)$ & & \\
\hline 2 & $7(21,21 \%)$ & & \\
\hline$\geq 3$ & $22(66,67 \%)$ & & \\
\hline PRESERVATIVE N (\%) & 0 & & \\
\hline Preservative-free & $28(84,85 \%)$ & & \\
\hline BAK & $5(15,15 \%)$ & & \\
\hline \multicolumn{4}{|l|}{$P Q+B A K$} \\
\hline \multicolumn{4}{|c|}{ ACTIVE INGREDIENT N (\%) } \\
\hline Prostaglandine & $28(84,85 \%)$ & & \\
\hline B-Blocker & $28(84,85 \%)$ & & \\
\hline CAI & $24(72,72 \%)$ & & \\
\hline AAA & $16(48,48 \%)$ & & \\
\hline \multicolumn{4}{|l|}{$D P C, \mu g / d$} \\
\hline Mean +/-SD & $1,89 \pm 0,88$ & & \\
\hline Minimum & 0,35 & & \\
\hline Maximun & 3,5 & & \\
\hline \multicolumn{4}{|c|}{$\mathrm{CPC}, \mu \mathrm{g} / \mathrm{d} \times$ treatment duration (days) } \\
\hline Mean +/-SD & $1670,15 \pm 1973,48$ & & \\
\hline Minimum & 53,20 & & \\
\hline Maximun & 10482 & & \\
\hline
\end{tabular}

In POAG group, we found a significant improvement in OSDI and NEI-VFQ25 scores from pre-surgery time to six months after NPDS (24.33 \pm 7.7 vs $15.06 \pm$ $6.93, \mathrm{p}<0.001$ and $61.78 \pm 13.20$ vs $74.42 \pm 11.97, \mathrm{p}<0.001$; respectively).

Treated subjects demonstrated a significant increase in the mean LTMH measured by K5M. This improvement is significant from the third postoperative month when compared with baseline values $(p \leq 0.025)$. When comparing the LTMH by FD-OCT, the improvement is observed six months after NPDS $(p=$ 
0.037). Before surgery, mean FD-OCT LTMH measurements in POAG eyes were significantly lower than control eyes ( $p=0.043)$; however, no significant differences were observed after six months $(p=0.062)$. Measurements of the LHTM by the K5M were not different between groups before and after NPDS ( $p$ $\geq 0.15$ ).

Before NPDS, the total and sector K5M-OH values in treated patients were significantly higher than in control group ( $p \leq 0.048$ ). These differences disappeared in the sixth month postoperative visit in temporal sectors and in the nasal-limbal sector $(p \geq 0.062)$. We observed a significant increase in total and sector values $\mathrm{K5M}-\mathrm{OH}$ in the first week post-NPDS compared to preoperative visit $(p<0.001)$; however, after six months, the total $\mathrm{OH}$ decreased significantly $(p=$ 0.003). Figure 1 shows an example of the evolution of $\mathrm{OH}$ in one of our patients.

In POAG group, we detected a significant improvement in NI-BUTf three months after NPDS ( $\mathrm{x} \leq 0.022$ ); nevertheless, the average NI-BUT (NI-BUTav) was significantly higher than baseline values during the first week $(\mathrm{p} \leq 0.048)$. Before surgery, NI-BUT measurements in glaucoma eyes were significantly lower than controls ( $p \leq 0.016)$; but no significant differences were observed in the sixth month visit $(p \geq 0.104)$. Figure 2 shows an example of the evolution of $\mathrm{NI}$ BUT in one of our patients. Table 2 summarizes the results of questionnaires (OSDI and NEI VQF25) and ocular surface parameters measured preoperatively, in the first week, first month, third and sixth month, after surgery in POAG and control group. Figure 3 shows the evolution of the OS parameters in the POAG group.

Table 2

Questionnaires (OSDI and NEI VQF25) and ocular surface parameters measured preoperatively (Mean \pm SD)

\begin{tabular}{|c|c|c|c|c|c|c|c|c|c|c|c|c|}
\hline \multirow[t]{2}{*}{ Parametres } & \multicolumn{8}{|c|}{ GLAUCOMA GROUP } & \multicolumn{4}{|c|}{ CONTROL GROUP } \\
\hline & $\begin{array}{l}\text { Pre- } \\
\text { NPDS }\end{array}$ & 1 week & $\mathbf{p}$ & $\begin{array}{l}1 \\
\text { month }\end{array}$ & $\mathbf{p}$ & $\begin{array}{l}3 \\
\text { months }\end{array}$ & $\mathbf{p}$ & $\begin{array}{l}6 \\
\text { months }\end{array}$ & $\mathbf{p}$ & Controls & $\begin{array}{l}\text { Baseline } \\
\text { versus } \\
\text { controls }\end{array}$ & $\begin{array}{l}6 \text { months } \\
\text { versus } \\
\text { controls }\end{array}$ \\
\hline OSDI (unit) & $\begin{array}{l}24.33 \pm \\
7.7\end{array}$ & & & & & & & $\begin{array}{l}15.06 \pm \\
6.93\end{array}$ & $\begin{array}{l}\mathrm{p}< \\
0.001 *\end{array}$ & $\begin{array}{l}6.24+ \\
3.345\end{array}$ & $p<0.001^{*}$ & $\mathrm{p}<0.001^{*}$ \\
\hline $\begin{array}{l}\text { NEI VQF25 } \\
\text { (unit) }\end{array}$ & $\begin{array}{l}61.78 \pm \\
13.20\end{array}$ & & & & & & & $\begin{array}{l}74.42 \pm \\
11.97\end{array}$ & $\begin{array}{l}\mathrm{p}< \\
0.001^{*}\end{array}$ & $\begin{array}{l}84.73 \pm \\
10.1\end{array}$ & $p<0.001^{*}$ & $p<0.001^{*}$ \\
\hline $\begin{array}{l}\text { LHTM FD- } \\
\text { OCT }(\mu \mathrm{m})\end{array}$ & $\begin{array}{l}258.85 \\
\pm 52.82\end{array}$ & $\begin{array}{l}282.65 \\
\pm 55.18\end{array}$ & $\begin{array}{l}p= \\
0.081^{*}\end{array}$ & $\begin{array}{l}277.35 \\
\pm 57.44\end{array}$ & $\begin{array}{l}p= \\
0.208^{*}\end{array}$ & $\begin{array}{l}281.19 \\
\pm 60.33\end{array}$ & $\begin{array}{l}p= \\
0.157^{*}\end{array}$ & $\begin{array}{l}296.19 \\
\pm 64.64\end{array}$ & $\begin{array}{l}\mathrm{p}= \\
0.037 *\end{array}$ & $\begin{array}{l}289.04 \\
\pm 51.95\end{array}$ & $p=0.043^{*}$ & $p=0.062^{\star}$ \\
\hline $\begin{array}{l}\text { LHTM K5M } \\
(\mathrm{mm})\end{array}$ & $\begin{array}{l}0.301 \pm \\
0.1\end{array}$ & $\begin{array}{l}0.421 \pm \\
0.17\end{array}$ & $\begin{array}{l}\mathrm{p}< \\
0.001 *\end{array}$ & $\begin{array}{l}0.341 \pm \\
0.02\end{array}$ & $\begin{array}{l}p= \\
0.053^{\star}\end{array}$ & $\begin{array}{l}0.364 \pm \\
0.11\end{array}$ & $\begin{array}{l}\mathrm{p}= \\
0.01 *\end{array}$ & $\begin{array}{l}0.352 \pm \\
0.15\end{array}$ & $\begin{array}{l}\mathrm{p}= \\
0.025^{\star}\end{array}$ & $\begin{array}{l}0.321 \pm \\
0.06\end{array}$ & $p=0.356^{\star}$ & $p=0.150^{\star}$ \\
\hline $\begin{array}{l}\text { TOH K5M } \\
\text { (score) }\end{array}$ & $\begin{array}{l}2.06 \pm \\
0.66\end{array}$ & $\begin{array}{l}2.71 \pm \\
0.79\end{array}$ & $\begin{array}{l}\mathrm{p}< \\
0.001 *\end{array}$ & $\begin{array}{l}1.90 \pm \\
0.70\end{array}$ & $\begin{array}{l}p= \\
0.076^{*}\end{array}$ & $\begin{array}{l}1.85 \pm \\
0.60\end{array}$ & $\begin{array}{l}p= \\
0.063^{*}\end{array}$ & $\begin{array}{l}1.69 \pm \\
0.61\end{array}$ & $\begin{array}{l}p= \\
0.003^{*}\end{array}$ & $\begin{array}{l}1.42 \pm \\
0.41\end{array}$ & $p<0.001^{*}$ & $p=0.039 *$ \\
\hline $\begin{array}{l}\text { NBH K5M } \\
\text { (score) }\end{array}$ & $\begin{array}{l}2.20 \pm \\
0.66\end{array}$ & $\begin{array}{l}2.86 \pm \\
0.80\end{array}$ & $\begin{array}{l}\mathrm{p}< \\
0.001 *\end{array}$ & $\begin{array}{l}2.13 \pm \\
0.75\end{array}$ & $\begin{array}{l}p= \\
0.538^{*}\end{array}$ & $\begin{array}{l}2.02 \pm \\
0.67\end{array}$ & $\begin{array}{l}p= \\
0.139 *\end{array}$ & $\begin{array}{l}1.83 \pm \\
0.65\end{array}$ & $\begin{array}{l}\mathrm{p}= \\
0.014^{*}\end{array}$ & $\begin{array}{l}1.54 \pm \\
0.42\end{array}$ & $p<0.001^{*}$ & $p=0.039 *$ \\
\hline $\begin{array}{l}\text { NLH K5M } \\
\text { (score) }\end{array}$ & $\begin{array}{l}1.85 \pm \\
0.65\end{array}$ & $\begin{array}{l}2.51 \pm \\
0.86\end{array}$ & $\begin{array}{l}p< \\
0.001 *\end{array}$ & $\begin{array}{l}1.52 \pm \\
0.64\end{array}$ & $\begin{array}{l}\mathrm{p}= \\
0.538^{*}\end{array}$ & $\begin{array}{l}1,59 \pm \\
0,66\end{array}$ & $\begin{array}{l}p= \\
0.457^{\star}\end{array}$ & $\begin{array}{l}1.52 \pm \\
0.66\end{array}$ & $\begin{array}{l}\mathrm{p}= \\
0.021 \text { * }\end{array}$ & $\begin{array}{l}1.05 \pm \\
0.43\end{array}$ & $p=0.001^{*}$ & $p=0.062^{*}$ \\
\hline $\begin{array}{l}\text { TBH K5M } \\
\text { (score) }\end{array}$ & $\begin{array}{l}1.92 \pm \\
0.65\end{array}$ & $\begin{array}{l}2.62 \pm \\
0.83\end{array}$ & $\begin{array}{l}p< \\
0.001 *\end{array}$ & $\begin{array}{l}1.81 \pm \\
0.63\end{array}$ & $\begin{array}{l}p= \\
0.220 *\end{array}$ & $\begin{array}{l}1.77 \pm \\
0.68\end{array}$ & $\begin{array}{l}\mathrm{p}= \\
0.212^{\star}\end{array}$ & $\begin{array}{l}1.67 \pm \\
0.64\end{array}$ & $\begin{array}{l}\mathrm{p}= \\
0.052^{\star}\end{array}$ & $\begin{array}{l}1.43 \pm \\
0.39\end{array}$ & $p<0.001^{*}$ & $p=0.068^{*}$ \\
\hline $\begin{array}{l}\text { TLH K5M } \\
\text { (score) }\end{array}$ & $\begin{array}{l}1.55 \pm \\
0.66\end{array}$ & $\begin{array}{l}2.3 \pm \\
0.89\end{array}$ & $\begin{array}{l}\mathrm{p}< \\
0.001 *\end{array}$ & $\begin{array}{l}1.42 \pm \\
0.72\end{array}$ & $\begin{array}{l}\mathrm{p}= \\
0.289 *\end{array}$ & $\begin{array}{l}1.27 \pm \\
0.54\end{array}$ & $\begin{array}{l}p= \\
0.016^{*}\end{array}$ & $\begin{array}{l}1.23 \pm \\
0.57\end{array}$ & $\begin{array}{l}p= \\
0.010 *\end{array}$ & $1.1 \pm 0.4$ & $p=0.048^{*}$ & $p=0.236^{\star}$ \\
\hline $\begin{array}{l}\text { NIBUTf } \\
\text { K5M (s) }\end{array}$ & $\begin{array}{l}5.96 \pm \\
4.15\end{array}$ & $\begin{array}{l}5.96 \pm \\
4.92\end{array}$ & $\begin{array}{l}\mathrm{p}= \\
0.999 *\end{array}$ & $\begin{array}{l}8.07 \pm \\
5.61\end{array}$ & $\begin{array}{l}\mathrm{p}= \\
0.100 *\end{array}$ & $\begin{array}{l}9.16 \pm \\
6.70\end{array}$ & $\begin{array}{l}p= \\
0.014^{*}\end{array}$ & $\begin{array}{l}8.47 \pm \\
5.19\end{array}$ & $\begin{array}{l}\mathrm{p}= \\
0.022^{*}\end{array}$ & $\begin{array}{l}8.87 \pm \\
5.29\end{array}$ & $p=0.016^{*}$ & $p=0.760 *$ \\
\hline $\begin{array}{l}\text { NIBUTav } \\
\text { K5M (s) }\end{array}$ & $\begin{array}{l}10.28 \pm \\
5.83\end{array}$ & $\begin{array}{l}12.84 \pm \\
5.76\end{array}$ & $\begin{array}{l}\mathrm{p}= \\
0.048^{*}\end{array}$ & $\begin{array}{l}14.92 \pm \\
4.53\end{array}$ & $\begin{array}{l}\mathrm{p}= \\
0.001 *\end{array}$ & $\begin{array}{l}16.53 \pm \\
4.38\end{array}$ & $\begin{array}{l}\mathrm{p}< \\
0.001 *\end{array}$ & $\begin{array}{l}15.72 \pm \\
3.99\end{array}$ & $\begin{array}{l}\mathrm{p}< \\
0.001 *\end{array}$ & $\begin{array}{l}13.81 \pm \\
5.31\end{array}$ & $p=0.013^{\star}$ & $p=0.104^{*}$ \\
\hline
\end{tabular}

Overall, meibography image study showed no significant changes in POAG group $(p>0.999)$. Significant higher scores were found in treated subjects when compared to control group, before and after surgery $(p \leq 0.006)$. Both, Table 3 and Fig. 4 show the evolution of meibography throughout the visits.

We observed a significant decrease in corneal and conjunctival staining scores (Oxford scale) six months after NPDS ( $<<0.001)$ in treated eyes. The percentages of eyes showing corneal and conjunctival staining at different time intervals are shown in Table 4 . Before surgery, $94 \%$ of POAG eyes (31 eyes) were classified as Oxford $\geq 1$; however, in the sixth month visit, only $18 \%$ of the treated eyes (six eyes) had fluorescein corneal staining ( $p<0.001)$. Similar

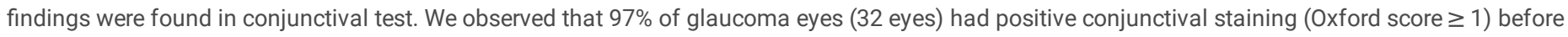
surgery; but this percentage significantly decreased to $42 \%$ (14 eyes) on the last visit $(p<0.001)$.

Table 3 Meibography measured with K5M. JENVIS grading scale. 


\begin{tabular}{|c|c|c|c|c|c|c|c|c|c|c|}
\hline \multirow[t]{3}{*}{ Parameters } & \multicolumn{10}{|c|}{ SUPERIOR MEIBOGRAPHY K5M JENVIS SCALE } \\
\hline & & & & & & \multicolumn{5}{|c|}{ INFERIOR MEIBOGRAPHY K5M JENVIS SCALE } \\
\hline & $\begin{array}{l}\text { Pre- } \\
\text { NPDS }\end{array}$ & 3 months & 6 months & Control & $\begin{array}{l}\text { P value POAG-control } \\
\text { before and } 6 \text { months }\end{array}$ & $\begin{array}{l}\text { Pre- } \\
\text { NPDS }\end{array}$ & 3 months & 6 months & Control & $\begin{array}{l}\text { P value } \\
\text { POAG- } \\
\text { control } \\
\text { before } \\
\text { and } 6 \\
\text { months }\end{array}$ \\
\hline GRADE 0 & $3.03 \%$ & $3.03 \%$ & $3.03 \%$ & $39.39 \%$ & & $12.12 \%$ & $15.2 \%$ & $24.2 \%$ & $48.48 \%$ & \\
\hline GRADE 1 & $39.39 \%$ & $39.39 \%$ & $39.39 \%$ & $57.58 \%$ & & $63.64 \%$ & $36.4 \%$ & $60.6 \%$ & $45.45 \%$ & \\
\hline GRADE 2 & $48.48 \%$ & $48.48 \%$ & $48.48 \%$ & $3.03 \%$ & & $18.18 \%$ & $42.45 \%$ & $15.2 \%$ & $6.06 \%$ & \\
\hline GRADE 3 & $9.09 \%$ & $9.09 \%$ & $9.09 \%$ & $0 \%$ & & $6.06 \%$ & $6.1 \%$ & $0 \%$ & $0 \%$ & \\
\hline$P$ value & & $\mathrm{p}>0.999 \star \star \star$ & $\mathrm{p}>0.999 * \star \star$ & & $\mathrm{p}<0.001^{\star \star}$ & & $p>0.999 \star \star \star$ & $p>0.999 \star \star \star$ & & $P=0.006^{* *}$ \\
\hline
\end{tabular}

** Mann- Whitney test.; ***Wilcoxon test;

A significantly greater proportion of POAG patients showed at least some fluorescein corneal and conjunctival staining before surgery compared to healthy control eyes $(p<0.001)$; however, no significant differences were observed during the last visit $(p \geq 0.254)$ (Table 4$)$. Figure 5 shows the improvement of conjunctival and corneal staining in the POAG group throughout the visits.

Table 4 Corneal and conjunctival stain (Oxford scale) results.

\begin{tabular}{|c|c|c|c|c|c|c|c|c|c|c|c|c|c|c|}
\hline \multirow[t]{2}{*}{ Parameters } & & \multicolumn{4}{|c|}{ CORNEAL STAINING OXFORD } & \multicolumn{5}{|c|}{ NASAL CONJUNCTIVAL STAINING OXFORD } & \multicolumn{4}{|c|}{ TEMPORAL CONJUNCTIVAL } \\
\hline & & $\begin{array}{l}\text { Pre- } \\
\text { NPDS }\end{array}$ & $\begin{array}{l}1 \\
\text { week }\end{array}$ & $\begin{array}{l}1 \\
\text { month }\end{array}$ & $\begin{array}{l}3 \\
\text { months }\end{array}$ & $\begin{array}{l}6 \\
\text { months }\end{array}$ & $\begin{array}{l}\text { Pre- } \\
\text { NPDS }\end{array}$ & $\begin{array}{l}1 \\
\text { week }\end{array}$ & $\begin{array}{l}1 \\
\text { month }\end{array}$ & $\begin{array}{l}3 \\
\text { months }\end{array}$ & $\begin{array}{l}6 \\
\text { months }\end{array}$ & $\begin{array}{l}\text { Pre- } \\
\text { NPDS }\end{array}$ & $\begin{array}{l}1 \\
\text { week }\end{array}$ & $\begin{array}{l}1 \\
\mathrm{~m}\end{array}$ \\
\hline \multirow{3}{*}{$\begin{array}{l}\text { POAG } \\
\text { GROUP }\end{array}$} & OXFORD & $6.1 \%$ & & & & & $3 \%$ & & & & & $3 \%$ & & \\
\hline & OXFORD & $93.9 \%$ & & $27.3 \%$ & $45.5 \%$ & & $97 \%$ & $0 \%$ & $3 \%$ & & $57.6 \%$ & $97 \%$ & & 6. \\
\hline & $\geq 1$ & & $100 \%$ & $72.7 \%$ & $54.5 \%$ & $18,18 \%$ & & $100 \%$ & $97 \%$ & $84.8 \%$ & $42.4 \%$ & & $100 \%$ & 9: \\
\hline $\begin{array}{l}\text { P value } \\
\text { POAG } \\
\text { group pre- } \\
\text { NPDS } \\
\text { and next } \\
\text { visits*** }\end{array}$ & & & $\begin{array}{l}p= \\
0.157\end{array}$ & $\begin{array}{l}p= \\
0.02\end{array}$ & $p<0.001$ & $p<0.001$ & & $\begin{array}{l}p= \\
0.317\end{array}$ & $p>0.999$ & $\begin{array}{l}p= \\
0.102\end{array}$ & $p<0.001$ & & $\begin{array}{l}p= \\
0.317\end{array}$ & $\begin{array}{l}\mathrm{p}: \\
0,\end{array}$ \\
\hline \multirow{2}{*}{$\begin{array}{l}\text { CONTROL } \\
\text { GROUP }\end{array}$} & OXFORD & $72.72 \%$ & & & & $72.72 \%$ & $78.79 \%$ & & & & $78.79 \%$ & $78.79 \%$ & & \\
\hline & $\begin{array}{l}\text { OXFORD } \\
\geq 1\end{array}$ & $27.27 \%$ & & & & $27.27 \%$ & $21.21 \%$ & & & & $21.21 \%$ & $21.21 \%$ & & \\
\hline $\begin{array}{l}\text { P value } \\
\text { POAG- } \\
\text { control ** }\end{array}$ & & $p<0.001$ & & & & $p=0.254$ & $p<0.001$ & & & & $p=0.66$ & $p<0.001$ & & \\
\hline
\end{tabular}

** Mann- Whitney test.; ***Wilcoxon test;

\section{Discussion}

The present study has found that patients with glaucoma had a significant improvement in OSD after NPDS. New non-invasive technologies such as K5M and FD-OCT could be useful tools to objectively study different parameters of the ocular surface that could help us to evaluate the OSD in glaucomatous eyes.

In this prospective study, all POAG patients were already in a combined hypotensive drug treatment with preservatives. Corneal neurotoxicity and tear film disorder are examples of side effects that have been already described in patients with glaucoma (26-28) due to the chronic use of IOP-lowering therapies. Therefore, the main idea of this study was to investigate the ocular surface changes after NPDS once anti-glaucoma eye drops were removed.

Portela et al.(29) and Ling et al.(30) have already described worst scores in OSDI and NEI-VQF25 questionnaires in glaucoma patients with hypotensive treatment. Similarly, before surgery, our study showed significant differences in the median OSDI and NEI-VFQ25 scores between POAG group and healthy controls. However, to our knowledge, there is no previous information about these questionnaires after NPDS. In the current study, we have observed a 
significant improvement in both tests during the sixth month after NPDS. Nevertheless, after surgery, we also found significant differences between groups. This may be due to the fact that $51.52 \%$ of patients have anti-glaucomatous treatment in the contralateral eye (bilateral POAG).

Our findings support literature evidence $(11,29,31-33)$, and the present study found that POAG eyes had a higher prevalence of OSD criteria than the healthy controls. Before NPDS, treated eyes, showed worse objective data regarding OH, LTMH,NIBUT and meibography evaluated by the Keratography 5M, worse FDOCT LTMH and higher Oxford scores on ophthalmologic examination than the healthy controls. However, we document a significant improvement in most of these parameters throughout the follow-up period. No significant differences were observed between the groups in the following parameters: FD-OCT LTMH, K5M LTMH, NIBUT (f and av values), corneal-conjunctival staining grades and in most $\mathrm{OH}$ sectors values six months after surgery. These findings could demonstrate an improvement in OSD after NPDS due to anti-glaucomatous drops withdrawn.

LTMH can be measured with both modern devices (FD-OCT and K5M) reducing the interpretation bias of subjective assessments. FD-OCT LTMH measurements have been proposed as a good diagnostic method for OSD; showing low variability $(34)$ good repeatability $(35,36)$ and a better reproducibility than the K5M (24). This could explain the difference in LTMH measurements when using the different devices. Therefore, these methods are not considered to be interchangeable.

Conjunctival hyperaemia is a common side effect described after the use of most topical anti-glaucoma medications (12, 31). Besides being a cosmetic problem, ocular hyperaemia may lead to poor treatment adherence. In recent studies, the Keratography $5 \mathrm{M}$ emerged as a reliable method to consistently score bulbar redness $(12,31,37)$. Previous studies $(12,31)$ have already described the relationship between conjunctival hyperaemia and anti-glaucoma medication using K5M. Our results are consistent with previous studies, we observed higher $\mathrm{K} 5 \mathrm{M} \mathrm{OH}$ in the glaucoma group. In our prospective study, a significant increase of $\mathrm{OH}$ was observed in the first week after surgery. It could be due to the surgical procedure itself and/or to the postoperative treatment with antiinflammatory drops. The $\mathrm{OH}$-sector scores were gradually restored to the baseline level, observing a significant improvement in most of $\mathrm{OH}$-sectors values during the sixth month. Significant group differences, disappeared by the sixth month in all the sectors except for the nasal bulbar one. Pérez-Bartolomé et al. (31) detected a greater impact of treatment burden on nasal hyperaemia scores than on the temporal quadrant ones. It could be explained by a longer contact time of the tear film in the nasal area, due to the normal spreading of the tear film across the ocular surface until it reaches the lacrimal punctum.

Previous studies have suggested that goblet cell secreting mucin 5AC, plays an important role in tear film stability (38) and several studies have reported toxic side effects for anti-glaucoma drugs on the conjunctiva, especially if preservatives are used $(12,39,40)$. Moreover, the reduction in goblet cell density and mucin 5AC could also be due to several glaucoma surgery factors such as: toxicity of MMC used during the surgery, damage of the conjunctival nerves and the limbus stem cells, inflammation or mechanical trauma produced by the surgery and post-operative topical medication (41-43). Zhong et al. (35) have recently investigated the influence of trabeculectomy and phacotrabeculectomy on the ocular surface in a 3-month retrospective study using Oculus Keratograph. They observed that both, NIBUTf and NIBUTav values, tended to improve in the first and third month after surgery; recovering baseline in the third month postoperatively. According to previous reports, goblet cell density did not return to preoperative level after three months cataract surgery (44), the same happened with mucin 5AC after phacotrabeculectomy (41). They propose that the improvement of the NI-BUT is related to the recovery of the levels of goblet cell density and mucin 5AC. In line with these findings, we also found NIBUTf and average values improvement in the third month after NPDS; additionally, not significant differences were observed in the sixth month compared to healthy eyes. Taking into account these results, parameters of ocular surface could not return to preoperative levels until three months post-NPDS.

Recent studies have suggested that trabeculectomy filtering bleb is a determining factor in the state of the ocular surface for at least half a year. It could cause dry eye by inducing meibomian gland loss, particularly when the bleb is avascular or contains numerous intraepithelial microcysts (45, 46). According to previous research, trabeculectomy filtering bleb morphology also affects tear film $(46,47)$ indicating that the higher filtering bleb would decrease TBUT and increase the corneal staining.

In the present study, no significant changes were observed in meibomian gland six months after NPDS. Furthermore, we documented a significant reduction in the percentage of glaucoma eyes with Oxford $\geq 1$ in the third month postoperatively, and no differences were recorded in corneal and conjunctival staining between groups in the last follow up visit. The different bleb morphology in NPDS and trabeculectomy could explain our results. (48, 49). Oh and colleagues found that eyes that underwent trabeculectomy had significantly higher, broader and lesser vascular blebs than eyes treated with deep sclerectomy. Also, eyes treated with trabeculectomy were more likely to show microcysts. Longer-term follow-up studies will be necessary to determine if deep sclerectomy bleb could cause more meibomian gland loss degrees.

Despite the significant findings, our study is subject to a number of limitations. First of all, the small sample size; which however has been enough to find significant differences, being the first study that uses non-invasive technologies (Keratograph and FD-OCT) to investigate OSD in glaucoma patients after NPDS. Second, the clinical ophthalmology examinations (corneal and conjunctival staining) are subjective, however, they were performed by just one ophthalmologist to avoid possible bias, and the comparison of keratitis was done using the Oxford scale, already published, obtaining therefore more reliable values. Finally, post-surgical steroids could improve ocular surface masking our results, nonetheless, the measurements of the third and sixth month should not be influenced by the treatment since it had already been completed.

In conclusion, although the association between anti-glaucoma eye drops and OSD has been extensively explored, to the best of our knowledge, this is the first study to compare different ocular surface parameters before and after NPDS. In addition, based on the objective parameters measured by Keratographs $5 \mathrm{M}$ and FD-OCT, we have reported a significant improvement of different variables such as OH-sectors, NI-BUT and LHTM after NPDS; as well as, lower cornealconjunctival fluorescein staining and better total scores on the OSDI and NEI-VFQ25 questionnaires than before surgery.

The current study demonstrates that the withdrawn of anti-glaucomatous topical treatment could improve the ocular surface six months after NPDS. In addition, NPDS may be considered as a good as surgical technique to restore the ocular surface, however further studies should be performed to corroborate 
our results.

\section{Abbreviations}

\begin{tabular}{|c|c|}
\hline BAK & Benzalkonium chloride \\
\hline CPC & Cumulative preservative concentration \\
\hline DES & Dry eye syndrome \\
\hline DPC & Daily preservative concentration \\
\hline FD-OCT & Fourier-domain optical coherence tomography \\
\hline IOP & Intraocular pressure \\
\hline K5M & Keratograph $5 \mathrm{M}$ \\
\hline LTMH & Lower tear meniscus height \\
\hline MGD & Meibomian gland dysfunction \\
\hline NBH & Nasal bulbar hyperemia \\
\hline NEI VFQ-25 & National Eye Institute Visual Functioning Questionnaire \\
\hline NI-BUT & Non-invasive tear film break-up time \\
\hline NIBUTav & Non-invasive tear film break-up time average \\
\hline NIBUTf & First non-invasive tear film break-up time \\
\hline NLH & Nasal limbal hyperemia \\
\hline NPDS & Nonpenetrating deep sclerectomy \\
\hline $\mathrm{OH}$ & Ocular hyperemia \\
\hline ON & Optic nerve \\
\hline OSD & Ocular Surface Disease \\
\hline OSDI & Ocular Surface Disease Index \\
\hline POAG & Primary Open Angle Glaucoma \\
\hline$P Q$ & Polyquaternium \\
\hline QoL & Quality of life \\
\hline SL & Slit lamp \\
\hline RS & Redness scores \\
\hline TLH & Temporal limbal hyperemia \\
\hline $\mathrm{TBH}$ & Temporal bulbar hyperemia \\
\hline TBUT & Tear film breakup time \\
\hline TOH & Total ocular hyperemia \\
\hline VA & Visual acuity \\
\hline
\end{tabular}

\section{Declarations}

\section{Disclosure/Conflicts of Interest}

Supported in part by Instituto de Salud Carlos III, "Red temática de Investigación Cooperativa, Proyecto RD16/0008. OftaRed: Prevención, detección precoz, tratamiento y rehabilitación de las patologías oculares"

\section{PRÉCIS}

The present study found that patients with anti-glaucomatous drugs had a significant improvement in ocular surface parameters, measured with K5M and FDOCT, after non-penetrating deep sclerectomy.

\section{References}

1. Weinreb RN, Aung T, Medeiros FA (2014 May) The pathophysiology and treatment of glaucoma: a review. JAMA 14(18):1901-1911 311( 
2. Quigley HA, Broman AT (2006) The number of people with glaucoma worldwide in 2010 and 2020. Br J Ophthalmol 90(3):262-267

3. Burgoyne CF, Downs JC, Bellezza AJ, Suh J-KF, Hart RT. The opticnerve head as a biomechanical structure: a new paradigm for understanding the role of IOP-related stress and strain in the pathophysiology of glaucomatous optic nerve head damage. 2005 Jan;24(1):39-73

4. Tuulonen A, Airaksinen PJ, Erola E, Forsman E, Friberg K, Kaila M et al. The Finnish evidence-based guideline for open-angle glaucoma. 2003 Feb;81(1):318

5. Heijl A, Leske MC, Bengtsson B, Hyman L, Bengtsson B, Hussein M (2002 Oct) Reduction of intraocular pressure and glaucoma progression: results from the Early Manifest Glaucoma Trial. Arch Ophthalmol 120(10):1268-1279

6. Chiselita D (2001 Apr) Non-penetrating deep sclerectomy versus trabeculectomy in primary open-angle glaucoma surgery. Eye (Lond) 15(Pt 2):197-201

7. The definition and classification of dry eye disease: report of the Definition and Classification Subcommittee of the International Dry Eye WorkShop (2007). Ocul Surf. 2007 Apr;5(2):75-92.

8. Mathews PM, Ramulu PY, Friedman DS, Utine CA, Akpek EK (2013) Evaluation of ocular surface disease in patients with glaucoma. Ophthalmology 120(11):2241-2248

9. Pisella P, Pouliquen P, Baudouin C (2002 Apr) Prevalence of ocular symptoms and signs with preserved and preservative free glaucoma medication. Br J Ophthalmol 86(4):418-423

10. Chawla A, McGalliard JN, Batterbury M (2007 Jun) Use of eyedrops in glaucoma: how can we help to reduce non-compliance? Acta Ophthalmol Scand 85(4):46

11. Miljanović B, Dana R, Sullivan DA, Schaumberg DA (2007 Mar) Impact of dry eyesyndrome on vision-related quality of life. Am J Ophthalmol 143(3):409415

12. Pérez-Bartolomé F, Martínez-de-la-Casa J, Arriola-Villalobos P, Fernández-Pérez C, Polo V, García-Feijoó J. Ocular surface disease in patients under topical treatment for glaucoma. Eur J Ophthalmol. 2017 Nov 8;27(6):694-704

13. Ram J, Gupta A, Brar GS, Kaushik S, Gupta A (2002 Aug) Outcomes of phacoemulsification in patients with dry eye. J Cataract Refract Surg 28(8):13861389

14. Leung EW, Medeiros FA, Weinreb RN (2008 Aug) Prevalence of ocular surface disease in glaucoma patients. J Glaucoma 17(5):350-355

15. Skalicky SE, Goldberg I, McCluskey P (2012 Jan) Ocular surface disease and quality of life in patients with glaucoma. Am J Ophthalmol 153(1):1-9

16. Valente C, lester M, Corsi E, Rolando M (2011 Jun) Symptoms and signs of tear film dysfunction in glaucomatous patients. J Ocul Pharmacol Ther 27(3):281-285

17. Lee S, Wong T, Chua J, Boo C, Soh Y, Tong L (2013 Oct) Effect of chronic anti- glaucoma medications and trabeculectomy on tear osmolarity. Eye (Lond) 27(10):1142-1150

18. Lam J, Wong TT, Tong L (2015 Jan) Ocular surface disease in posttrabeculectomy/mitomycin C patients. Clin Ophthalmol 29:9:187-191

19. Craig JP, Nichols KK, Akpek EK, Caffery B, Dua HS, Joo CK et al. TFOS DEWS II Definition and Classification Report.Ocul Surf. 2017 Jul;15(3):276-283

20. Barber L, Khodai O, Croley T, Lievens C, Montaquila S, Ziemanski J et al (2018 Sep) Dry eye symptoms and impact on vision-related function across International Task Force guidelines severity levels in the United States. BMC Ophthalmol 29(1):260 18(

21. Schiffman RM, Christianson MD, Jacobsen G, Hirsch JD, Reis BL (2000 May) Reliability and validity of the Ocular Surface Disease Index. Arch Ophthalmol 118(5):615-621

22. Simao LM, Lana-Peixoto MA, Araujo CR et al (2008) The Brazilian version of the 25-Item National Eye Institute Visual Function Questionnaire: translation, reliability and validity. Arq Bras Oftalmol 71:540-546

23. Arita R, Itoh K, Inoue K, Amano S. Noncontact infrared meibography to document age-related changes of the meibomian glands in a normal population. Ophthalmology. 2008 May;115(5):911-5

24. Arriola-Villalobos P, Fernández-Vigo JI, Díaz-Valle D, Peraza-Nieves JE, Fernández-Pérez C, Benítez-del-Castillo JM (2015 Aug) Assessment of lower tear meniscus measurements obtained with Keratograph and agreement with Fourier-domain optical-coherence tomography. Br J Ophthalmol 99(8):11201125

25. Lemp MA, Bron AJ, Baudouin C, del Castillo JMB, Geffen D, Tauber J et al (2011 May) Tear osmolarity in the diagnosis and management of dry eye disease. Am J Ophthalmol 151(5):792-798

26. Clouzeau C, Godefroy D, Riancho L, Rostène W, Baudouin C, Brignole-Baudouin F (2012) Hyperosmolarity potentiates toxic effects of benzalkonium chloride on conjunctival epithelial cells in vitro. Mol Vis 18:851-863

27. Pellinen P, Huhtala A, Tolonen A, Lokkila J, Mäenpää J, Uusitalo H (2012 Feb) The cytotoxic effects of preserved and preservative-free prostaglandin analogs on human corneal and conjunctival epithelium in vitro and the distribution of benzalkonium chloride homologs in ocular surface tissues in vivo. Curr Eye Res 37(2):145-154

28. Paimela T, Ryhänen T, Kauppinen A, Marttila L, Salminen A, Kaarniranta K. The preservative polyquaternium-1 increases cytoxicity and NF-kappaB linked inflammation in human corneal epithelial cells. Mol Vis. 2012;18:1189-96Ra S, Ayaki M, Yuki K, Tsubota K, Negishi K. Dry eye, sleep quality, and mood status in glaucoma patients receiving prostaglandin monotherapy were comparable with those in non-glaucoma subjects.PLoS One. 2017 Nov 27;12(11):e0188534

29. Portela RC, Fares NT, Machado LF, São Leão AF, de Freitas D et al (2018 Sep) Evaluation of Ocular Surface Disease in Patients With Glaucoma: Clinical Parameters, Self-report Assessment, and Keratograph Analysis. J Glaucoma 27(9):794-801 
30. Ling TE, Othman K, Yan OP, Rashid RA, Tet CM, Yaakob A et al. Evaluation of Ocular Surface Disease in Asian Patients with Primary Angle Closure. Open Ophthalmol J. 2017 Feb 28;11:31-39

31. Pérez Bartolomé F, Martínez de la Casa JM, Arriola Villalobos P, Fernández Pérez C, Polo V, Sánchez Jean R et al (2018) Ocular Redness Measured with the Keratograph 5M in Patients Using Anti- Glaucoma Eye Drops. Semin Ophthalmol 33(5):643-650

32. Feldman RM (2003 Feb) Conjunctival hyperemia and the use of topical prostaglandins in glaucoma and ocular hypertension. J Ocul Pharmacol Ther 19(1):23-35

33. Baffa Ldo P, Ricardo JR, Dias AC, Módulo CM, Braz AM, Paula JS et al. Tear film and ocular surface alterations in chronic users of antiglaucoma medications. Arq Bras Oftalmol. 2008 Jan- Feb;71(1):18-21

34. Bitton E, Keech A, Simpson T, Jones L (2007 Sep) Variability of the analysis of the tear meniscus height by optical coherence tomography. Optom Vis Sci 84(9):903-908

35. Zhong S, Zhou H, Chen X, Zhang W, Yi L (2019 Apr) Influence of glaucoma surgery on the ocular surface using oculus keratograph. Int Ophthalmol $39(4): 745-752$

36. Tittler EH, Bujak MC, Nguyen P, Zhang X, Li Y, Yiu SC, Huang D

37. Between-grader repeatability (2011 Sep-Oct) of tear meniscus measurements using Fourier-domain OCT in patients with dry eye. Ophthalmic Surg Lasers Imaging 42(5):423-427

38. Feldman RM (2003 Feb) Conjunctival hyperemia and the use of topical prostaglandins in glaucoma and ocular hypertension. J Ocul Pharmacol Ther 19(1):23-35

39. Ablamowicz AF, Nichols JJ (2016 Jul) Ocular Surface Membrane-Associated Mucins. Ocul Surf 14(3):331-341

40. Souchier M, Buron N, Lafontaine PO, Bron AM, Baudouin C, Creuzot-Garcher C (2006 Nov) Trefoil factor family 1, MUC5AC and human leucocyte antigenDR expression by conjunctival cells in patients with glaucoma treated with chronic drugs: could these markers predict the success of glaucoma surgery? Br J Ophthalmol 90(11):1366-1369

41. de Jong C, Stolwijk T, Kuppens E, de Keizer R, van Best J (1994 Apr) Topical timolol with and without benzalkonium chloride: epithelial permeability and autofluorescence of the cornea in glaucoma. Graefes Arch Clin Exp Ophthalmol 232(4):221-224

42. Oh T, Jung Y, Chang D, Kim J, Kim H (2012 Mar) Changes in the tear film and ocular surface after cataract surgery. Jpn J Ophthalmol 56(2):113-118

43. Pflugfelder SC, Liu Z, Monroy D, Li DQ, Carvajal ME, Price-Schiavi SA et al (2000 May) Detection of sialomucin complex (MUC4) in human ocular surface epithelium and tear fluid. Invest Ophthalmol Vis Sci 41(6):1316-1326

44. Liu W, Li H, Lu D, Liang J, Xing X, Liu A et al. The tear fluid mucin 5AC change of primary angle-closure glaucoma patients after short-term medications and phacotrabeculectomy. Mol Vis. 2010 Nov 9;16:2342-6

45. Amar N, Labbé A, Hamard P, Dupas B, Baudouin C (2008 Jul) Filtering blebs and aqueous pathway an immunocytological and in vivo confocal microscopy study. Ophthalmology 115(7):1154-1161

46. Sagara H, Sekiryu T, Noji H, Ogasawara M, Sugano Y, Horikiri H (2014 Jul) Meibomian gland loss due to trabeculectomy. Jpn J Ophthalmol 58(4):334341

47. Ji H, Zhu Y, Zhang Y, Li Z, Ge J, Zhuo Y (2016 Mar) Dry Eye Disease in Patients with Functioning Filtering Blebs after Trabeculectomy. PLoS One 31(3):e0152696 11(

48. Neves Mendes CR, Hida RY, Kasahara N (2012 Apr) Ocular surface changes in eyes with glaucoma filtering blebs. Curr Eye Res 37(4):309-311

49. Kerr NM (2017 Sep) Differences in bleb morphology between trabeculectomy and deep sclerectomy. Clin Exp Ophthalmol 45(7):675-676

50. Oh LJ, Wong E, Lam J, Clement Cl (2017 Sep) Comparison of bleb morphology between trabeculectomy and deep sclerectomy using a clinical grading scale and anterior segment optical coherence tomography. Clin Exp Ophthalmol 45(7):701-707

\section{Figures}
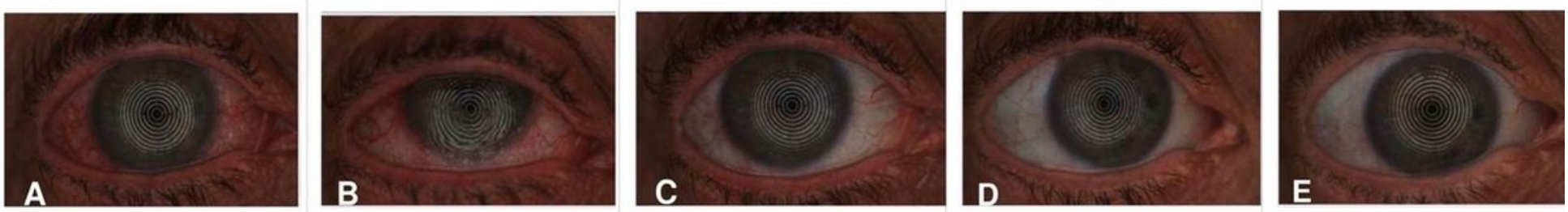

Figure 1. Ocular hyperaemia: A: Pre-surgery; B: 1 week; C: 1 month; D: 3 months; E: 6 months

\section{Figure 1}

Ocular hyperaemia: A: Pre-surgery; B: 1 week; C: 1 month; D: 3 months; E: 6 months 

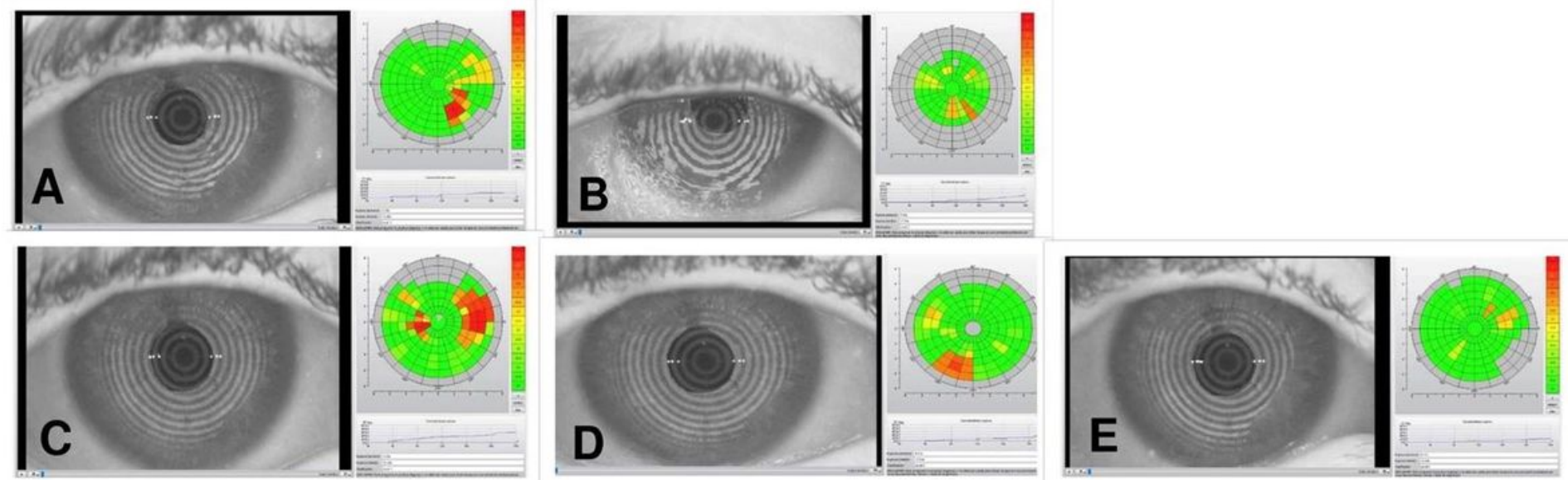

Figure 2. NIBUT: A: Pre-surgery; B: 1 week; C: 1 month; D: 3 months; E: 6 months

Figure 2

NIBUT: A: Pre-surgery; B: 1 week; C: 1 month; D: 3 months; E: 6 months

\section{OCULAR SURFACE PARAMETERS IN POAG GROUP}
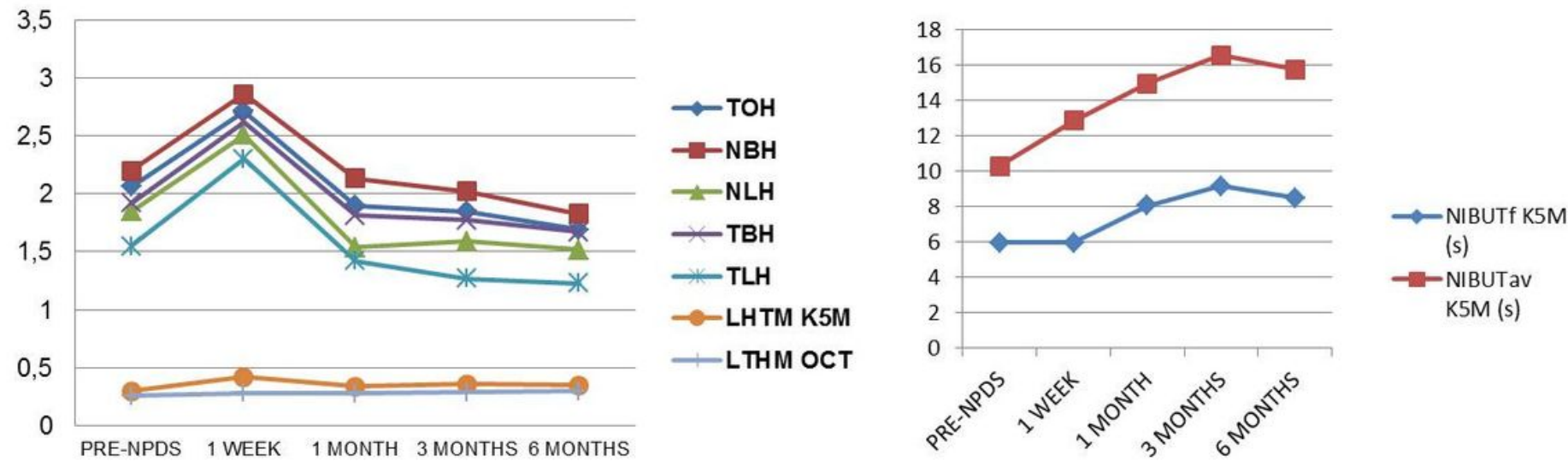

Figure 3: Ocular surface parameters measured in POAG Group. LHTM Lower tear meniscus height; TOH: total ocular hyperemia; NBH: nasal bulbar hyperemia; NLH: nasal limbal hyperemia, TBH: temporal bulbar hyperemia; TLH: temporal limbal hyperemia; NIBUTf: First non-invasive tear film break-up time; NIBUTav: Noninvasive tear film break-up time average

Figure 3

Ocular surface parameters measured in POAG group.

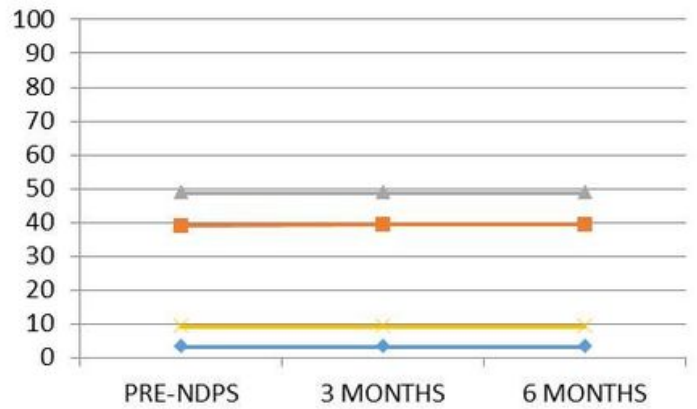

SUPERIOR
MEIBOGRAPHY
K5M JENVIS
SCALE POAG
GROUP

$\rightarrow-$ GRADE 0
$\rightarrow-$ GRADE 1
- GRADE 2
- GRADE 3

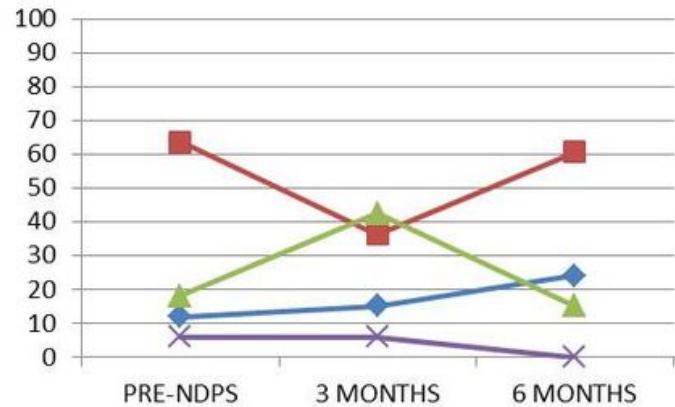

INFERIOR MEIBOGRAPHY K5M JENVIS SCALE POAG GROUP

$\leadsto$ GRADE 0

-GRADE 1

$\longrightarrow$ GRADE 2

$\because$ GRADE 3

Figure 4: Meibography K5M Jenvis scale in POAG group 
Figure 4

A: Meibography K5M JENVIS scale in POAG group. B:Corneal and conjunctival staining Oxford scale in POAG group.
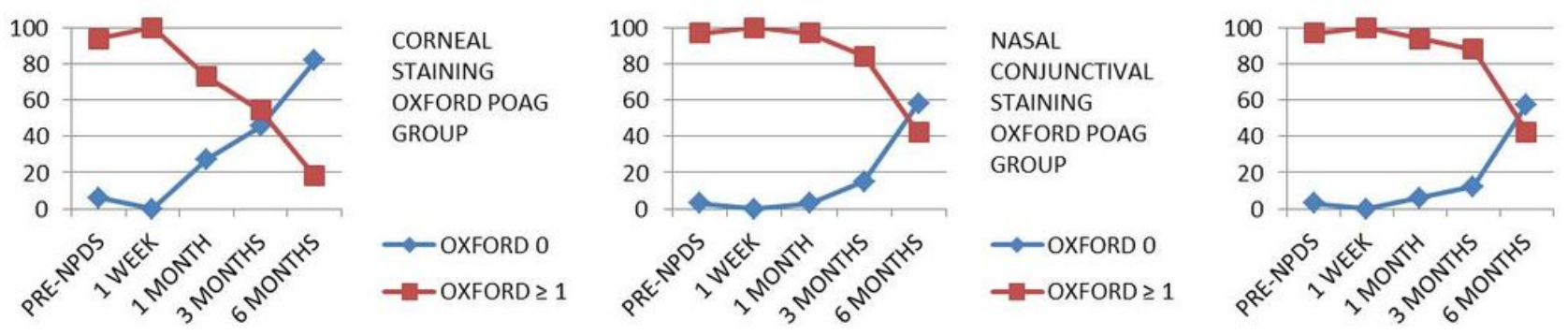

TEMPORAL

CONJUNCTIVAL

STAINING

OXFORD POAG

GROUP

$\rightarrow$ OXFORD 0

- - $-O X F O R D \geq 1$

Figure 5: Corneal and conjunctival staining Oxford scale in POAG group

\section{Figure 5}

Corneal and conjunctival staining Oxford scale in POAG group. 